\title{
A NOTE ON EXTREMAL PROBLEMS FOR CERTAIN CLASSES OF ANALYTIC FUNCTIONS
}

\author{
W. E. KIRWAN
}

1. Introduction. Let $\mathfrak{F}$ denote a normal compact family of functions $f(z)$ which are analytic in $|z|<1$ and which satisfy $f(0)=0$ and $f^{\prime}(0)=1$. Most extremal problems for such a family of functions may be expressed in the form

$$
\max _{f \in \mathcal{F}}(\min ) \operatorname{Re} F\left(f(c), \cdots, f^{(n)}(c), c\right)
$$

where $|c|<1$ and $F\left(w_{0}, \cdots, w_{n+1}\right)$ is analytic on $U_{f \in \mathcal{F}}(f(c), \cdots$, $\left.f^{(n)}(c), c\right)$. For certain families $\mathfrak{F}$ it is possible to describe analytically a subclass of $\mathcal{F}$ which contains the extremal functions of (1.1) for arbitrary $F\left(w_{0}, \cdots, w_{n+1}\right)$. Variational techniques afford a natural device for solving such a problem. However, in using this technique it has usually been found necessary to assume that if $f(z)$ is an extremal function for (1.1),

$$
\sum_{k=0}^{n}\left|F_{k}\left(f(c), \cdots, f^{(n)}(c), c\right)\right| \neq 0 .
$$

(See $[1, \S 3$, Theorems 1, 2, 3, and 4]; [5, Theorem 3.1] and [7, Theorems 2 and 3]; see also [2, Theorem 1] and [6, Theorem 1] where the condition is assumed but not explicitly stated.) The purpose of this note is to show that in many cases, including those just cited, the restriction (1.2) is unnecessary.

2. For the remainder of the paper we assume that the family $\mathcal{F}$ has the property that if $f(z) \in \mathcal{F}, f(t z) / t \in \mathcal{F}$ for each fixed $t$ in $0<|t| \leqq 1$. A family of functions which satisfies this condition will be called rotationally invariant. Since $\mathcal{F}$ is compact and $\lim _{t \rightarrow 0} f(t z) / t=z$, a rotationally invariant family of functions contains the function $e(z)=z$. Examples of rotationally invariant families are the class $\delta$ of functions $f(z)$ which are analytic and univalent in $|z|<1$ and which satisfy $f(0)=0$ and $f^{\prime}(0)=1$, and the subclasses of $S$ consisting of the starlike functions, the convex functions and the close-to-convex functions (see [3, p. 169] and [4, p. 221] for definitions).

THEOREM. Let $\mathcal{F}$ denote a rotationally invariant family of functions and assume $F\left(w_{0}, \cdots, w_{n+1}\right)$ is analytic and nonconstant on

Received by the editors April 20, 1966. 
$\bigcup_{f \in \mathcal{F}}\left(f(c), \cdots, f^{(n)}(c), c\right)$ where $|c|<1$. If $f(z)$ is an extremal function for (1.1), then

$$
\sum_{k=0}^{n}\left|F_{k}\left(f(c), \cdots, f^{(n)}(c), c\right)\right| \neq 0
$$

Proof. First we show the assumption $F\left(w_{0}, \cdots, w_{n+1}\right)$ is nonconstant on $\bigcup_{f \in \mathcal{F}}\left(f(c), \cdots, f^{(n)}(c), c\right)$ implies that the function $e(z)=z$ is not an extremal function for (1.1). Assume the contrary. Let $f(z) \in \mathcal{F}$ and let $f_{t}(z)=f(t z) / t$ with $|t| \leqq 1$ (the function $e(z)=z$ corresponds to $t=0)$. Since $\mathcal{F}$ is rotationally invariant, $f_{t}(z) \in \mathcal{F}$ and

$$
F\left(f_{t}(c), \cdots, f_{t}^{(n)}(c), c\right)=F\left(f(t c) / t, \cdots, t^{n-1} f^{(n)}(t c), c\right) .
$$

Define $G(t)=F\left(f_{t}(c), \cdots, f_{t}^{(n)}(c), c\right) . G(t)$ is clearly analytic in $|t| \leqq 1$. The assumption that $e(z)$ is an extremal function enables us to write

$$
\begin{aligned}
\operatorname{Re} G(0) & =\operatorname{Re} F(c, 1,0, \cdots, 0, c) \\
& \geqq \operatorname{Re} F\left(f_{t}(c), \cdots, f_{t}^{(n)}(c), c\right) \\
& =\operatorname{Re} G(t)
\end{aligned}
$$

which in view of the analyticity of $G(t)$ implies $G(t)$ is constant in $|t| \leqq 1$. Thus

$$
\operatorname{Re} G(0)=\operatorname{Re} G(1)=\operatorname{Re}\left(f(c), \cdots, f^{(n)}(c), c\right) .
$$

Since $f(z)$ is an arbitrary function in $\mathcal{F},(2.1)$ implies $F\left(w_{0}, \cdots, w_{n+1}\right)$ is constant on $U_{f \in \mathcal{F}}\left(f(c), \cdots, f^{(n)}(c), c\right)$ contrary to the hypothesis.

Now let $f(z)$ denote an extremal function for (1.1) and assume

$$
\sum_{k=0}^{n}\left|F_{k}\left(f(c), \cdots, f^{(n)}(c), c\right)\right|=0 .
$$

Let $K$ denote the disc $|1-w| \leqq 1$. For fixed $w$ in $K, f_{w}(z)=f[(1-w) z]$ $/(1-w)$ defines a function in $\mathcal{F}$ with $f_{w}^{(k)}(z)=(1-w)^{k-1} f^{(k)}[(1-w) z]$. The function $H(w)$ defined by

$$
H(w)=F\left(f_{w}(c), \cdots, f_{w}^{(n)}(c), c\right)
$$

is analytic in $K$ and in particular at $w=0$. Expanding $H(w)$ in a Taylor series at $w=0$ we have

$$
\begin{aligned}
H(w) & =F\left(f_{w}(c), \cdots, f_{w}^{(n)}(c), c\right) \\
& =F\left(f(c), \cdots, f^{(n)}(c), c\right)+\sum_{k=2}^{\infty} B_{k} w^{k}
\end{aligned}
$$


where the assumption (2.2) assures that there is no first degree term in $w$. This expansion is valid in a disc $D$ with center $w=0$.

For $w$ in $K \cap D, f_{w}(z) \in \mathcal{F}$. Since $f(z)$ is an extremal function for (1.1),

$$
\operatorname{Re} \sum_{k=2}^{\infty} B_{k} w^{k} \leqq 0, \quad(w \in K \cap D) .
$$

Given any value of $\theta,-\pi / 2<\theta<\pi / 2$, there exists an $r(\theta)>0$ such that when $0<r<r(\theta), w=r e^{i \theta} \in K \cap D$. For such $w$ the preceding inequality may be written $\operatorname{Re}\left\{r^{2} e^{2 i \theta} B_{2}+o\left(r^{2}\right)\right\} \leqq 0$.

This implies $\operatorname{Re} e^{i \alpha} B_{2} \geqq 0$ for $-\pi<\alpha<\pi$. Thus $B_{2}=0$ and

$$
F\left(f_{w}(c), \cdots, f_{w}^{(n)}(c), c\right)=F\left(f(c), \cdots, f^{(n)}(c), c\right)+\sum_{k=3}^{\infty} B_{k} w^{k} .
$$

A similar argument shows $B_{3}=0$ and indeed for all $k, B_{k}=0$. Hence

$$
F\left(f_{w}(c), \cdots, f_{w}^{(n)}(c), c\right)=F\left(f(c), \cdots, f^{(n)}(c), c\right)
$$

for $w$ in $K \cap D$ and consequently in $K$. Taking $w=1$ in (2.3), we have

$$
F(c, 1,0, \cdots, 0, c)=F\left(f(c), \cdots, f^{(n)}(c), c\right) .
$$

Thus the function $e(z)=z$ is an extremal function for (1.1) which by the first part of the proof is impossible. The contradiction arose from the assumption (2.2). Therefore $\sum_{k=0}^{n}\left|F_{k}\left(f(c), \cdots, f^{(n)}(c), c\right)\right| \neq 0$ and the proof is complete.

We remark that with only slight modification the same proof yields a similar result for the class of functions $p(z)$ analytic in $|z|<1$ which satisfy $p(0)=1$ and $\operatorname{Re} p(z)>0$ for $|z|<1$. Thus in addition to the theorems in [1] and [2], the above result is also applicable to the previously listed theorems of [5], [6] and [7].

\section{REFERENCES}

1. G. M. Goluzin, $A$ variational method in the theory of analytic functions, Trans. Amer. Math. Soc. 18 (1961), 8-14.

2. J. A. Hummel, $A$ variational method for starlike functions, Proc. Amer. Math. Soc. 9 (1958), 82-87.

3. W. Kaplan, Close-to-convex functions, Michigan Math. J. 1 (1952), 169-185.

4. Z. Nehari, Conformal mapping, McGraw-Hill, New York, 1952.

5. J. Pfaltzgraff, Extremal problems and coefficient regions for analytic functions represented by a Stieltjes integral, Trans. Amer. Math. Soc. 115 (1965), 270-282.

6. M. S. Robertson, Extremal problems for analytic functions with positive real part and applications, Trans. Amer. Math. Soc. 106 (1963), 236-253.

7. K. Sakaguchi, A variational method for functions with positive real part, J. Math. Soc. Japan 16 (1964), 287-296. 